\title{
Aerodynamics of an aerofoil in transonic ground effect: Methods for blowdown wind- tunnel scale testing
}

\author{
G. Doig and T. J. Barber \\ g.doig@unsw.edu.au \\ School of Mechanical and Manufacturing Engineering \\ The University of New South Wales \\ Sydney, Australia

\section{A. J. Neely} \\ School of Aerospace, Civil and Mechanical Engineering \\ The University of New South Wales at the Australian Defence Force Academy \\ Canberra, Australia
}

\section{D. Myre}

Aerospace Engineering Department

The United States Naval Academy

Maryland, USA

\begin{abstract}
Experimental aerodynamic testing of objects in close ground proximity at high subsonic Mach numbers is difficult due to the construction of a transonic moving ground being largely unfeasible. Two simple, passive methods have been evaluated for their suitability for such testing in a small blowdown wind tunnel: an elevated ground plane, and a symmetry (or mirror-image) approach. The methods were examined using an unswept wing of RAE2822 section, with experiments and Reynolds-Averaged Navier Stokes CFD used synergistically to determine the relative merits of the techniques. The symmetry method was found to be a superior approximation of a moving ground in all cases, with mild discrepancies observed only at the lowest ground clearance. The elevated ground plane was generally found to influence the oncoming flow and distort the flowfield between the wing and ground, such that the method provided a less-satisfactory match to moving ground simulations compared to the symmetry technique.
\end{abstract}

Paper No. 3642. Manuscript received 22 October 2010, revised version received 21 June 2011, accepted 13 September 2011. 


\section{NOMENCLATURE}

$\begin{array}{ll}c & \text { chord } \\ C_{L} & \text { coefficient of lift } \\ C_{D} & \text { coefficient of drag } \\ C_{P} & \text { coefficient of pressure } \\ D & \text { drag force } \\ h & \text { minimum height above ground plane } \\ I & \text { turbulent intensity } \\ L & \text { lift force } \\ \mathrm{M} & \text { local Mach number } \\ \mathrm{M}_{\infty} & \text { freestream Mach number } \\ V_{\infty} & \text { freestream velocity } \\ x & \text { distance from leading edge } \\ \alpha & \text { angle-of-attack } \\ \rho_{\infty} & \text { freestream density }\end{array}$

\subsection{INTRODUCTION}

Experimental aerodynamic testing at high subsonic Mach numbers for situations such as extremely low-flying military aircraft, magnetic-levitation launch vehicles, or even future high speed trains, presents the significant challenge of ground representation. A moving ground which operates at such speeds is essentially unfeasible, at least not without considerable expense and complexity. For this reason, an acceptable alternative is required, and the choices made dictate the nature of the interaction between the ground and strong pressure gradients or shock waves produced by the body in question. Military facilities such as rocket sleds can be used ${ }^{(1)}$, but these are expensive and access can be severely restricted. The use of an elevated ground has been proposed $^{(2)}$, but can produce unacceptable interference, particularly if the model is small in relation to the ground boundary layer which forms. Indeed, such issues of scale make the use of any specific ground apparatus a considerable problem (though scale issues are certainly not a problem unique to ground effect studies).

With a lack of acceptable and cost-effective wind-tunnel testing techniques, increasing emphasis is being placed on the use of computational fluid dynamics (CFD) as the primary or sole design tool, particularly for private, unconventional vehicles such as land speed record cars $^{(3)}$. The use of CFD (with conventional Reynolds-Averaged Navier-Stokes (RANS) modelling), with its persistent difficulties in modelling general turbulent structures and boundary layer transition as well as the multi-scale complexities of shock wave interactions (and reflections, in ground effect), requires careful experimental validation. The identification of a suitable wind-tunnel method is therefore important, and this paper investigates two relatively simple, passive approaches: an elevated ground plane, and a 'mirror-image' symmetry model.

The correct ground boundary condition for all ground effect testing in a wind tunnel is a moving ground, conventionally achieved by having a belt travelling at the freestream flow velocity. For larger tunnels and models it is often approximated more crudely with an elevated ground plane. This produces a boundary layer which, though undesirable, can be minimised with a combination of suction and blowing. In the earliest days of ground effect research, it was also shown analytically that a symmetry (or mirror-image) method can be a good approximation for the desired ground boundary ${ }^{(4)}$. 
Numerical studies have highlighted the difference between stationary, slip wall, symmetry and moving ground boundaries for a lifting NACA 4412 aerofoil in ground effect ${ }^{(5)}$. The symmetry method was seen to produce near-identical results to a moving ground simulation up until very low ground clearances $(h / c<0 \cdot 05)$, at which point a recirculation ahead of the wing at the ground plane produced inaccurate results. An analogy was drawn between this observed effect and a vortex pair in a potential flow. The symmetry method did, however, correctly predict a slight drop off in lift at the lowest clearances, as was observed for the moving ground cases due to the extreme acceleration of flow under the wing. The CFD was conducted at a freestream Mach number of 0.32 with the flow treated as incompressible, although at this Mach number and at such low ground clearances, one would expect significant flow compressibility to be present.

The symmetry method is not commonly used experimentally for ground effect studies, though is often used in a different context for shock reflection studies ${ }^{(9,10)}$. The method was implemented for studies by NASA in the $1960 \mathrm{~s}^{(6)}$. It was asserted that tests with a symmetry wing setup with endplates, relatively far from wall interference, had produced results which agreed well with data for wings actually moving over a ground. Wind-tunnel experiments with NACA 4 -series aerofoils have also been conducted firstly with a fixed ground plane ${ }^{(7)}$, where the boundary layer was found to merge with that of the aerofoil to distort results at low clearances as in other studies ${ }^{(8)}$, and subsequently with a moving ground. The latter study also examined the low clearance lift-loss at small angles of incidence, as previously observed by others ${ }^{(5)}$, and discussed the importance of the geometry of the curvature of the lower surface in producing this lower surface suction effect. However, these studies were conducted at Reynolds numbers of approximately $3 \times 10^{5}$, equating to freestream velocities of $30 \mathrm{~ms}^{-1}\left(\mathrm{M}_{\infty}<0 \cdot 1\right)$.

Discrete suction to remove or minimise a ground boundary layer on a wind-tunnel floor or elevated ground is often implemented in the automotive industry, but would be a more complicated proposition at transonic Mach numbers. These techniques would also have the inherent problem of introducing small-scale disturbances into the sensitive flow in the region of interest between the object and the ground, particularly in critical flows where various waveforms would result at extremely low ground clearances.

The symmetry and elevated ground methods have already been investigated by the authors using schlieren photography and numerical analysis for fully supersonic (Mach 2.4) flows in a blowdown wind-tunnel with a model of a projectile ${ }^{(11)}$. The multiple shock/ground interactions and reflections in that study were influenced by the presence of the ground boundary layer of the elevated ground method, and mild waves generated by the ground plane leading edge influenced the oncoming flow to a slight degree. The symmetry method, providing the models were identical and well aligned, provided a virtually identical match to simulations involving a moving ground, and therefore was deemed to be an excellent means to investigate such flows in a small blowdown tunnel.

The current study extends the investigation into the potentially more complex transonic regime, where additional complications can be forseen: any ground boundary layer (even in the instance of a moving ground, where the pressure field around an object causes a ground boundary layer to form) will be thicker than in supersonic flows, and the potential for unsteady flows is greatly increased; the buffet boundary in particular may be sensitive to the ground representation and its influence. The elevated ground cannot help but affect the speed and direction of the oncoming flow, and the additional blockage presented by having an elevated ground or symmetry model is a significant consideration in limiting the maximum Mach number (or Reynolds number) one can reliably achieve in a conventional blowdown facility. In addition, any asymmetry present in the symmetry method apparatus will cause the flow to adjust 


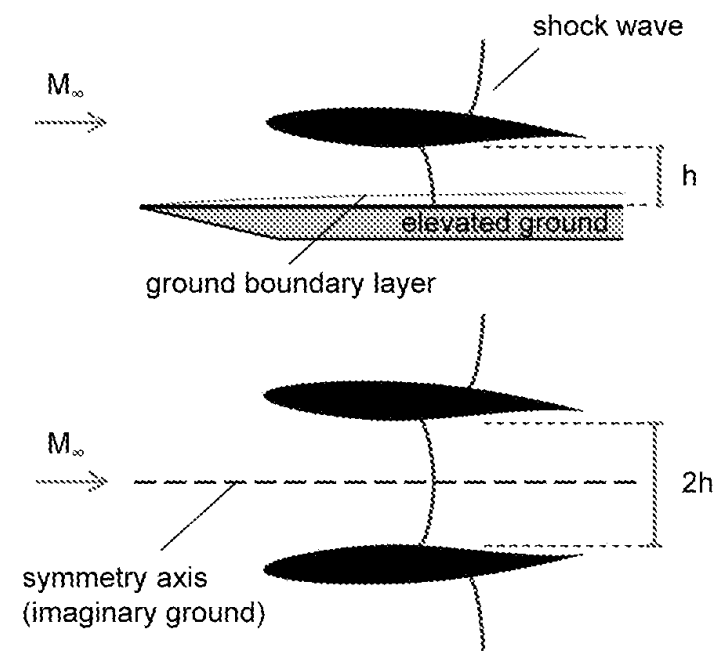

Figure 1. Schematic of the elevated ground and symmetry methods as applied to the RAE2822 aerofoil.

to a state which would provide incorrect flow over the entirety of both wings. The two methods are outlined for a critical flowfield around an aerofoil in Fig. 1.

Preliminary wind-tunnel experiments using a simple RAE2822 section in symmetry and elevated ground configurations, relying on discrete pressure tappings, have been carried out at mid-subsonic Mach numbers. These tests were supplemented by numerical simulations of the experiments in order to better quantify the relative merits of each method, and enhance understanding of the limitations of the wind-tunnel tests. The research therefore involves a constructive relationship between experimental testing and CFD; in essence using the CFD to first help design and then confirm the effectiveness of the experiments, and using the experiments to validate the computational approach so it can be used for further understanding the flows investigated.

\subsection{EXPERIMENTAL METHOD}

The transonic blowdown wind tunnel of the United States Naval Academy in Annapolis, Maryland, features a test section of cross-sectional area $0.0418 \mathrm{~m}^{2}$, with dimensions of 0.2057 $\times 0.2032 \mathrm{~m}\left(8 \cdot 1^{\prime \prime} \mathrm{h}, 8^{\prime \prime} \mathrm{w}\right)$, and incorporates optional adjustable porous ceiling and floor plates which vent/suck air to/from a plenum chamber of nominally-atmospheric conditions if required. Mach number is varied as a function of stagnation pressure coupled with the use of a downstream choke; values for both were selected based on existing calibration data which had previously allowed a determination of settings producing the greatest consistency of Mach number flow in the test section. The porous floor and ceiling were completely closed for all of the experiments described here in order to facilitate more reliable CFD modelling of the test conditions with simple boundaries, as is commonly recommended (but seldom implemented) for any code validation of this nature ${ }^{(12)}$.

The turbulent intensity, $I$, of the flow in the test section was investigated: an average value of $I$ of $0 \cdot 16 \%$ was obtained by evaluation of data from runs at three Mach numbers with the test section empty, and was relatively consistent with a variation of no more than $0.02 \%$ from run to run. At this value, the tunnel was deemed to provide an acceptably low level of turbulence. 


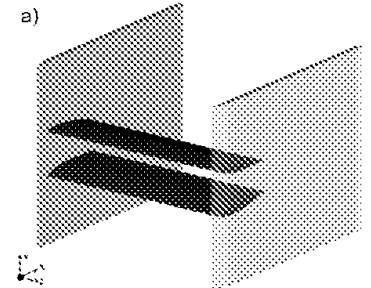

(a)

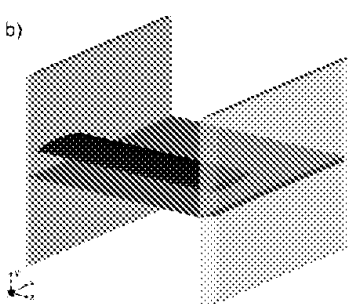

(b)

Figure 2. The elevated ground method (a) and symmetry method (b)

\subsection{Apparatus, technique and measurement techniques}

The elevated ground plane and symmetry method apparatus for the tests are shown schematically in Figs 2(a) and 2(b) respectively. The wing section was an RAE2822 section, the co-ordinates of which can be found in the original AGARD report for code validation from $1979^{(13)}$. The decision was made to use this geometry not just because of the obvious possibility for further validation of the numerical methods employed, but in order to enable the recontextualisation of this well-known aerofoil, such that its changed characteristics when in ground effect would stand in contrast to its familiar 'freeflight' traits. The chord was $58 \mathrm{~mm}$ (close to $10 \%$ of the scale used in the AGARD experiments), with the trailing-edge purposely blunted (from the original chord of $60 \mathrm{~mm}$ ) after manufacture to ensure a consistent and robust edge. A relatively small aspect ratio of three was necessary to allow for internal instrumentation. Hypodermic pipe from the pressure tappings exited the wings at the side, into vinyl tubing in gaps in the steel endplates which were necessary as modifications to the tunnel walls were not possible to route the vinyl away from the wings. The internal diameter of the hypodermic tube was $0.5 \mathrm{~mm}$, which was considerable in relation to the dimensions of the wing, but staggering the tappings diagonally across the middle third of the wing ensured that downstream disturbance and interference would be minimal.

Due to the very small internal volume of the wings, a decision was made to have a set of tappings for the upper surface on one wing, and tappings for the lower surface on the other wing. Given that the wings were close to identical due to the highly-repeatable manufacturing process, this effectively provided one complete set of 16 upper and lower pressure readings per symmetry test. 'Wing B' featured seven tappings on the upper surface. Chordwise tappings were confined to the middle third of the wing, where it was expected the flow would be largely twodimensional. Three other tappings were arranged at $x / c=0 \cdot 17$, with spanwise $z / b$ locations of $0 \cdot 10,0 \cdot 30$, and $0 \cdot 48$ from the endplate. These were designed to provide a simple way of assessing the two-dimensionality of the flow in the region of the chordwise pressure tappings - this will be discussed further in the following section. The lower surface of wing A featured a more detailed arrangement of nine tappings, also in a diagonally-staggered fashion. Based on early numerical analysis of possible configurations, there was an expectation that a lowersurface shock invoked by close ground proximity could be produced at approximately $\mathrm{M}_{\infty}=0 \cdot 6$. Therefore the increased emphasis on the lower surface for pressure readings was a reasonable choice in sacrificing resolution on the upper surface. A tapping at the leading edge was introduced to provide a stagnation pressure reading. 
The elevated ground was tapped at 12 locations, clustered towards the point at which the wing's maximum thickness would occur and thus where the strongest gradients would likely be observed, and staggered diagonally across the middle $1 / 3$ of the structure as with the wings. The leading edge of the wing was $0.015 \mathrm{~m}$ behind the leading edge of the elevated ground.

The wings, endplates and rear sting mount strut together comprised a test section blockage of $12 \%$, and with the porous ceiling and floor closed off. This blockage value was based on frontal area for two wings in symmetry configuration, at $0^{\circ}$ angle-of-attack. The blockage was slightly higher $(12 \cdot 8 \%)$ for the wings at $6^{\circ}$ angle-of-attack, and slightly lower $(9 \%)$ for the tests involving the ground plane, as the rear sting mount strut was largely 'hidden' from the oncoming flow by the ground plane itself, and the ground plane was thinner than the wing. Blockages in all cases were relatively high, but unavoidably so in order to introduce the instrumentation with a secondary aim of keeping the Reynolds number as high as possible. The influential effect of this will be discussed in the following section.

The rig was designed such that four ground clearances and two angles of attack were possible. The readings from the pressure ports on the wings and ground were taken as timeaveraged data of 100 samples at $10 \mathrm{~Hz}$ with a calculated standard deviation. The sample rate was not sufficient to accurately capture the higher frequencies associated with oscillatory shock behaviour, and thus it would be misleading to treat the data obtained as truly representative of any transient behaviour. If a large standard deviation was obtained, it was taken to be indicative in itself of transient behaviour, and this insight was clarified with subsequent simulation.

A simple RMS approach was used to determine error in the calculation of pressure coefficients at each discrete point measurements were taken from. A variety of other sources of error would be influential, but because of insufficient time at the facility to make the necessary tests to quantify them thoroughly, these are not included in the error bars with which the results are presented. Standard deviations of the velocity, freestream static pressure, and discrete static pressure measurements were derived from experimental data, and errors implicit in the calculation of freestream density stemmed from established limitations in the instruments used to measure temperature, as well as the known fluctuations in stagnation pressure. It is estimated that all other sources of error would potentially be an order of magnitude less important, following regular calibration procedures. General repeatability of the experiments proved to be excellent: the maximum deviation in average Mach number for any desired value was determined to be \pm 0.003 for experiments conducted at several stagnation pressures and atmospheric conditions.

It is regrettable that boundary layer transition on the wings was not fixed for the experiments, and thus the location at which it occurred across the range of variables tested was not determinable. Numerical analysis indicated that transition is unlikely to have occurred more than $0 \cdot 3 c$ from the leading edge, and fully turbulent simulations provide an acceptable comparison (see Section 3.0).

General manufacturing tolerances for all model parts were nominally $0.05 \mathrm{~mm}$, however due to the nature of the machining process, extensive sanding of the wings was required and therefore a true tolerance of $\sim 0 \cdot 2 \mathrm{~mm}$ is more likely for the most crucial parts, based on measurements made with digital callipers. This represents $2.5 \%$ of the wing thickness, or a little under half a percent of the chord length, which becomes significant at very low ground clearances. Additionally, a standard error in angle-of-attack of $\pm 0 \cdot 1^{\circ}$ was estimated. While these geometric facets are not factored into the error bars presented in the graphs of pressure coefficient, they are almost certainly at least a minor factor in the occasional discrepancies between CFD and experimental results, particularly at the lowest ground clearances where the influence would be greatest. 


\subsection{NUMERICAL METHOD}

As numerical analysis was used in the study to confirm and quantify the effectiveness of the experimental method in the absence of an actual moving ground experiment for comparison, the computational approach had to be extensively validated as an integral part of the study. Aspects of the experiments not immediately obvious from the tests themselves were revealed by the CFD, such as the effect of the endplates in practice, the nature of shock oscillations, and the influence of boundary layer transition. A commercial finitevolume Reynolds-Averaged Navier Stokes solver, Fluent $6 \cdot 3^{(15)}$, was used to generate the results. A pressure-based, coupled solver was applied to obtain flow solutions, and convergence criteria were deemed to be met not only when the mass and momentum scaled-residual errors ceased to change by more than approximately $0.01 \%$ over 1,000 continued iterations, but also when the aerodynamic forces on the body ceased to change by more than $0 \cdot 01 \%$ over 1,000 further iterations (for the steady-state simulations). All cases were run in 64-bit double precision using a second order node-based upwind discretisation scheme. A standard three-coefficient Sutherland viscosity model was applied $^{(16)}$. Comparison solutions with the density-based solver available produced very similar results (typically $<0 \cdot 2 \%$ difference in force coefficients), but required considerably more iterations in most cases and therefore to reduce the computational expense the pressure-based solver was preferred.

Four cases from the experimental test programme have been chosen for discussion and are outlined in Table 1. They have been selected to provide a range of flowfields to necessitate thorough validation of both the experimental method and the accompanying simulations. They do not necessarily represent the 'best' matches with CFD data obtained from the whole test programme. Rather, they highlight the accuracy and limitations of the simulations (and experiments) in a handful of situations of varying complexity, yet the general conclusions which hold across other scenarios. All the results presented for numerical validation in this section come from symmetry method experiments unless stated otherwise, and deal with the full three-dimensional geometry emulating the experimental setup, as opposed to simpler two-dimensional results presented subsequently.

Case 0 is a shock-free 'freeflight' case (i.e. no ground plane), as measured as close to the centre of the tunnel as possible, taken as a reference case to estimate transition and wall effects.

Case 1 features no shock waves in the test section, though the air between the wing and ground is accelerated to near-sonic local conditions (approximately $\mathrm{M}_{3}=0 \cdot 97$ ). CFD of Case 1 was run as transient until a nominally steady-state solution was obtained, to ensure damping of any persistent unsteadiness due to the early stages of the simulation forming supersonic flow.

Case 2 presents the wing at an incidence of $6^{\circ}$, promoting a strong shock wave towards the leading-edge on the upper surface. The simulations were again run as transient separation at the wing/endplate junction was observed, causing mild unsteadiness in the flowfield and a periodic spanwise disturbance - the shock wave itself was not exhibiting buffet behaviour.

Case 3 presents the most complex flowfield observed in the tunnel: a mildly-oscillating shock on the upper surface, with an accompanying shock on the lower surface oscillating in a regular, larger-scale motion. Significant supersonic flow is also present around the endplate leading-edges on the wing side, causing recurring separation. 
Table 1

Cases used for comparisons, and associated flow conditions

$\begin{array}{llccl} & \mathbf{M}_{\infty} & \boldsymbol{\alpha}_{\mathbf{o}} & \boldsymbol{h} / \boldsymbol{c} & \text { Comments } \\ \text { Case 0 } & 0 \cdot 59 & 0 & \text { n/a } & \text { 'free-flight' (no ground), shock-free, steady flowfield } \\ \text { Case 1 } & 0 \cdot 53 & 0 & 0 \cdot 13 & \text { shock-free, steady flowfield } \\ \text { Case 2 } & 0 \cdot 63 & 6 & 0 \cdot 23 & \text { upper surface shock, unsteady flowfield } \\ \text { Case 3 } & 0 \cdot 70 & 0 & 0 \cdot 27 & \begin{array}{l}\text { upper surface shock almost stationary at symmetry plane, } \\ \text { regularly-oscillating lower surface shock }\end{array}\end{array}$

\subsection{Mesh and boundary considerations}

The meshes and turbulence models assessed are first examined purely as comparisons against the experimental data obtained, which at this stage were treated as nominally two-dimensional pressure distributions at the wing mid-span. Three-dimensional characteristics of the flowfield are assessed subsequently. All results in this section were generated with the Spalart-Allmaras turbulence model, the selection of which will be discussed in the following section.

A portion of a sample mesh is presented in Fig. 3, for an elevated ground model. For all cases, the simulations were run with an $x y$-symmetry plane at the wing mid-span. For the cases involving the symmetry method, a $z x$-plane symmetry plane was used at the imaginary ground plane, and for cases involving the elevated ground, that apparatus was modelled in full. The endplate leading-edge angles matched the wind-tunnel model. The sting mount strut at the rear of the endplates, which also provided sufficient rigidity to the assembly, was included in early simulations to assess its effect on the flowfield and to determine if it was necessary to include it in all numerical cases, but was not found to exert significant influence. The sidewall boundary layer was assumed to begin at the test section inlet - no information on the wall boundary layers was available for input into the numerical model. Given the relatively small gap between the endplate and the sidewall boundary layer, this assumption may have had ramifications for the extent of separation around the endplate leading-edge seen at higher Mach numbers.

Fully-structured multi-block coarse, standard and fine meshes were constructed. The presence of unsteady effects in cases two and three meant that the coarse mesh was not formally assessed, as it would not have had sufficient cell density to resolve the complexity of these flowfields. The standard mesh featured 374 cells around the wing in the chordwise sense,

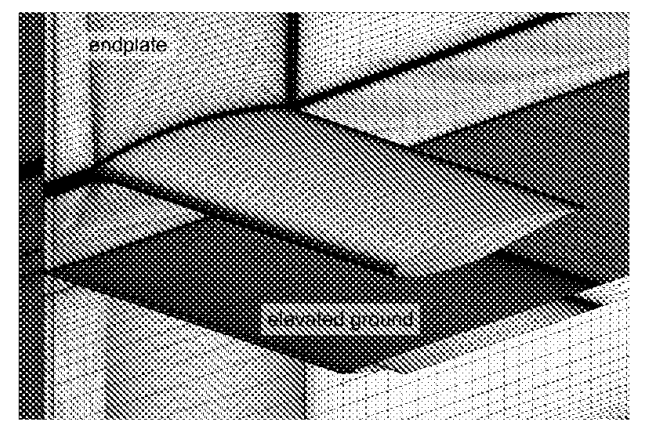

(a)
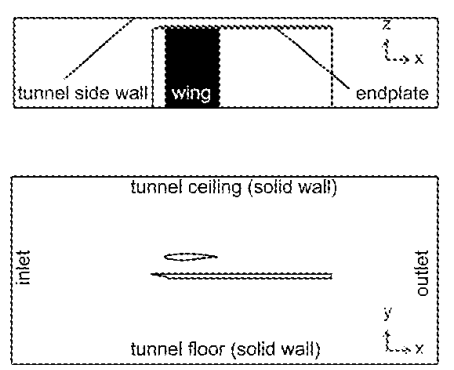

(b)

Figure 3. An example of typical mesh on the wing, endplate and ground plane for elevated ground configuration, and planar schematics of the whole domain (to scale). 
clustered towards the leading and trailing edges, and 40 spanwise cells on the half-wing, clustered towards the junction with the endplate. The $y^{+}$values for attached flow on the wing, elevated ground (if used) and endplate varied between 0.1 and 3 depending on the Reynolds number and flow conditions. A typical symmetry method mesh contained approximately $3.9 \times$ $10^{6}$ to $4 \cdot 2 \times 10^{6}$ cells, depending on the ground clearance. With the elevated ground, the mesh contained approximately $5.9 \times 10^{6}$ to $6.4 \times 10^{6}$ cells.

The fine mesh featured 55 spanwise cells on the half-wing, and 568 cells around the wing in the chordwise sense, with the total number of cells in the region of $7.9 \times 10^{6}$ to $8.3 \times 10^{6}$. The wake region of the wing was slightly refined, but otherwise the other blocks of the grid were left the same as the standard mesh.

Figure 4(a) shows a comparison between the standard and fine meshes for the shock-free Case 1. The meshes produced almost identical pressure distributions at the mid-span symmetry plane; they both exhibited reasonable agreement with experimental data, though the pressure across the upper surface was overestimated by a consistent amount. The pressure tapping behind the lower surface suction peak is not well-matched by the CFD. The simulations indicated that the peak local Mach number here was approximately Mach 0.97 , such that any minor change to the flow conditions experienced by the wing may have caused a local pocket of supersonic flow to form. It is possible that this is why the experimental pressure recovery is slightly further downstream than that predicted by the CFD, though the experimental distribution indicates lower pressure over the upper surface than that seen in the numerical result, and this implies that more flow was directed over the upper surface in the experiment such that the attainment of a locally supersonic Mach number on the lower surface would be less likely. Mesh comparisons for Case 2 (not shown) produced similar negligibly-different results, such that the standard mesh was preferred in both these cases.

Figure 4(b) shows time-averaged pressure distributions (over 3 regular oscillation cycles) for the two meshes for Case 3. Again, both meshes match the experimental results satisfactorily, albeit slightly underestimating the upper surface pressure beyond $x / c=0 \cdot 4$, and overestimating the lower surface pressure drop over the forward half of the chord. The lower surface shock wave oscillated considerably, and therefore it is in this region that differences between the two meshes are observed. The standard mesh provides a closer match to the experimental readings in this location, which indicates that the higher level of smearing in the shock region as it moved

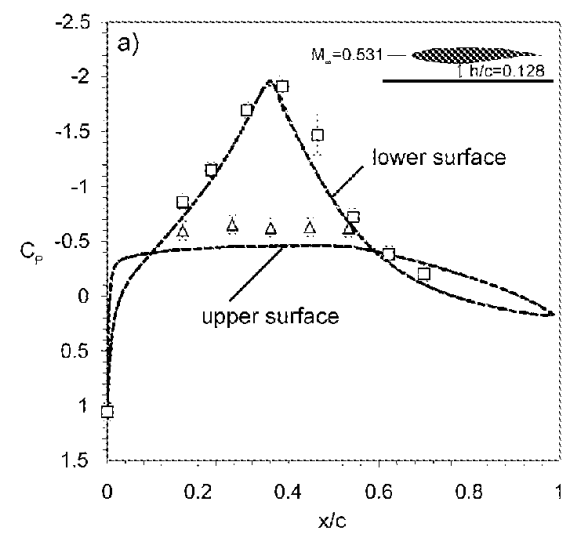

(a)

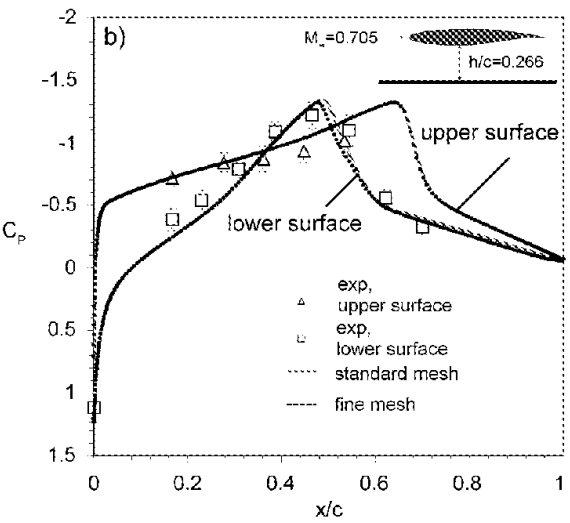

(b)

Figure 4. Pressure distribution at the mid-span (symmetry plane) for (a) Case 1, and (b) a time-averaged plot for Case 3 , for standard and fine meshes. 
with time may have provided a more fortuitous result than the mesh which physically resolved the waves more effectively. Without the means to effectively pinpoint the source of the discrepancy, the standard mesh was deemed to be of an acceptable quality to produce results within the margin of experimental error, and was used for all subsequent simulations.

\subsection{Turbulence modelling}

As the wings were not equipped with transition strips, free transition occurred at an indeterminate point along the chord. In order to assess the applicability of a fully-turbulent CFD solution in comparison to the experiments, some simulations were run to understand the effect of transition on the results which might be obtained.

Cases 0 and 3 were selected, presenting the simplest and most complex tunnel flowfields, respectively. Two runs for Case 0 were conducted - one fully turbulent with the Spalart-Allmaras model ${ }^{(17)}$, and one with the $k-k-1-\omega$ model which has proven effective for transonic flowfields with free transition ${ }^{(18)}$. The contour plot in Fig. 5(a) suggests that transition would have occurred at approximately $x / c=0 \cdot 4$, with a contamination from the endplate which has only a minor spanwise effect that appears to be highly sensitive to the mesh. The pressure distributions presented in Fig. 5(b) indicate that this has had a relatively small effect on the overall characteristics of the wing. One must bear in mind, though, that although the geometry of the wind-tunnel apparatus was faithfully reproduced and the inlet turbulence characteristics matched, the oncoming flow in the CFD is extremely smooth in time compared to the wind-tunnel flow. Therefore in the experiment, the transition line would be continually changing, and would have also been affected by the surface roughness of the wing ${ }^{(14)}$, as well as the aforementioned uncertainties regarding the sidewall boundary layer. However, based on the pressure distributions of Case 0 , it can be surmised that a fully-turbulent simulation is a reasonable approximation of the flowfield, with the differences generally within the existing margin of experimental error.

Investigations by Sudani et al. suggest that having transition occur this far back on the wing, particularly when there is an oscillating shock wave, can have a strong influence on the characteristics of that shock ${ }^{(14)}$. Therefore three simulations were made with Case 3 ; with transition

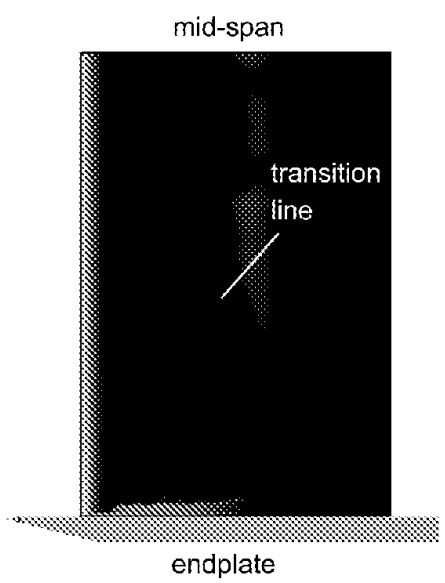

(a)

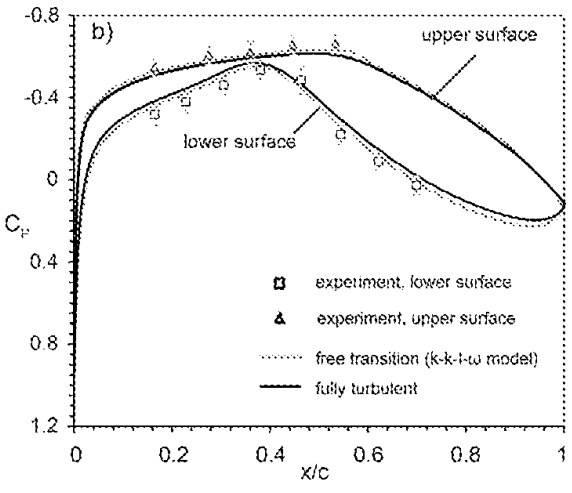

(b)

Figure 5. (a) Contours of skin friction for the lower wing surface, Case 0 (no ground effect); (b) pressure distribution at the mid-span plane, comparison to experiment for free transition and fully turbulent CFD. 


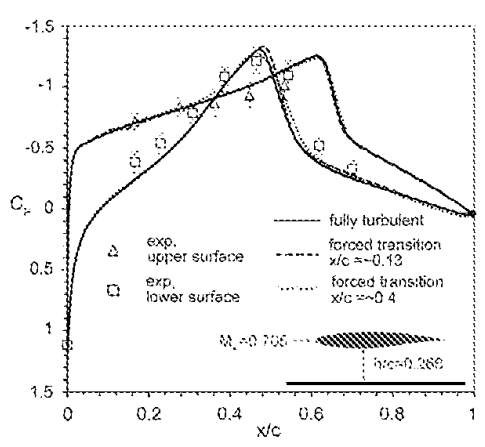

Figure 6. Time-averaged ressure distributions at the mid-span symmetry plane, Case 4, free transition and fully turbulent CFD. numerically tripped at $x / c=0 \cdot 13$, at $x / c=0 \cdot 40$, and fully-turbulent. Time-averaged pressure distributions, representing three full cycles of the wing $C_{L}$ as the upper and lower shocks oscillated, are presented in Fig. 6 and, encouragingly, present only minor differences despite the large variation in transition. The case with transition at $x / c=0.4$ is closest to the experimental results by a small margin, which would indicate that this transition location is closest to that which occurred in the wind-tunnel.

Based on these two sets of close comparisons and in the absence of a reliable measurement of the actual point of transition, fully turbulent simulations were run for all remaining cases. It would have been difficult to justify using the $k-k-1-\omega$ model for all simulations - apart from considerably longer run times, the model did not perform with adequate stability in the presence of strongly separated flow. As a result, the Spalart-Allmaras and Realisable $k-\varepsilon$ models ${ }^{(19)}$ were the only ones used for comparison in the remainder of the study.

Example pressure distributions obtained with the two models are presented for Cases 1,2 and 3 in figures 7 to 9 . Case 1 comparisons show both models matching the experiment acceptably, although both overpredicted the upper surface pressure as with the mesh comparisons, and failed to match to the tapping beyond the lower surface pressure peak. Given that both models and both meshes tested produce this result, it is increasingly likely that experimental factors not well reproduced in the numerical model are responsible. The Realisable model predicted barelysupersonic flow at the suction peak on the lower surface, indicating the sensitivity of the flowfield to modelling choices at critical points on the Mach number scale. The ground pressures obtained during tests with the elevated ground plane are also presented, and highlight good agreement with experiment while exhibiting negligible differences between the models.

The pressure distributions obtained for Case 2, in Fig. 9, indicate a more severe discrepancy in results between models. The Spalart-Allmaras model predicts a small region of shockseparated flow on the upper surface from approximately $x / c=0 \cdot 165$ to $0 \cdot 205$, whereas the Realisable $k$ - $\varepsilon$ model predicts the shock further back along the chord, and with no significant separation of the flow. The Spalart-Allmaras simulation provides notably better agreement with the experimental results for the two tappings upstream and downstream of the shock, though both models again overestimate the lower surface pressure readings. The ground pressure distribution is predicted with marginally better accuracy by the SA model.

The clearest difference between the models appears in the Case 3 comparison in Fig. 9. Here, the upper surface shock is significantly more smeared in the time-averaged plot with the Realisable model than with the Spalart-Allmaras model, as a result of a much greater chordwise movement of the shock there, with additional periodic flow separation from the moving shock foot. The lower surface distributions are in closer agreement, although again a greater level of smearing is present in the Realisable plot. Unlike with the upper surface shock, this is apparently due to a greater level of diffusion with the two-equation model, as the extent of shock movement is very similar compared to that of the Spalart-Allmaras simulation. The latter appears to better predict the average shock location more satisfactorily, and provides a better match in the pressure recovery region as a consequence. The ground pressures from the tapping closest to 


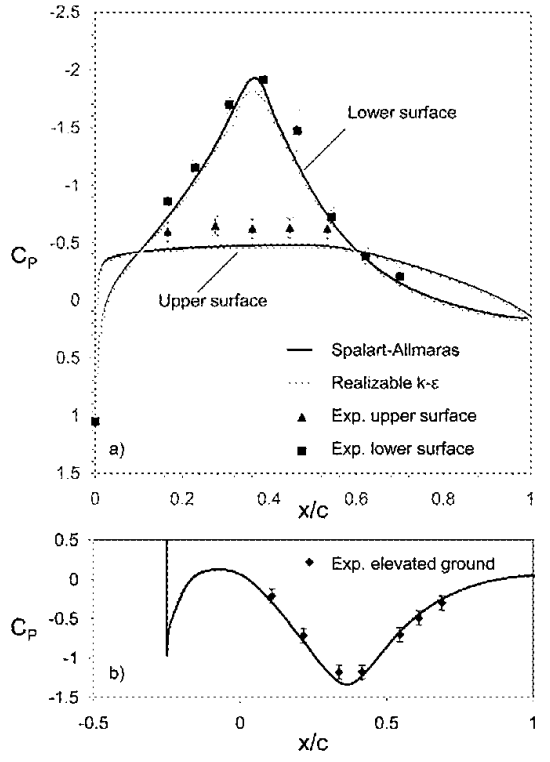

Figure 7. Chordwise pressure distributions with turbulence model comparisons to experiment for Case 1, for the semi-span (symmetry plane) on the wing (top), and elevated ground plane (bottom).
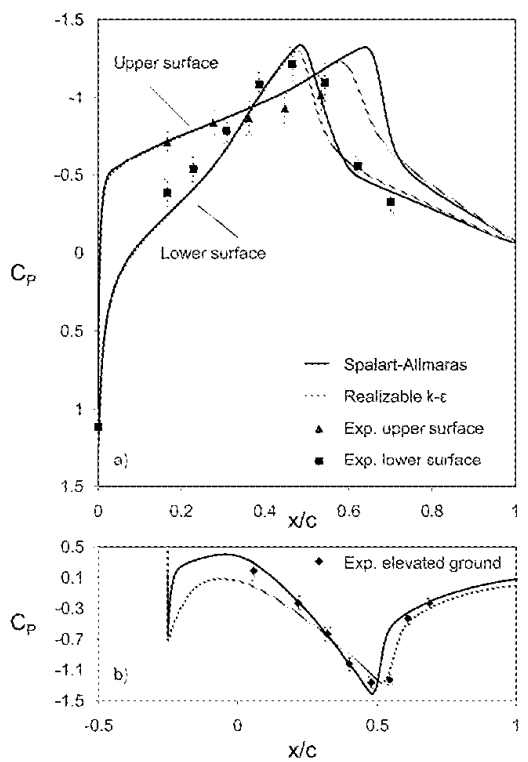

Figure 9. Chordwise pressure distributions with turbulence model comparisons to experiment for Case 3, for the semi-span (symmetry plane) on the wing (top), and elevated ground plane (bottom).

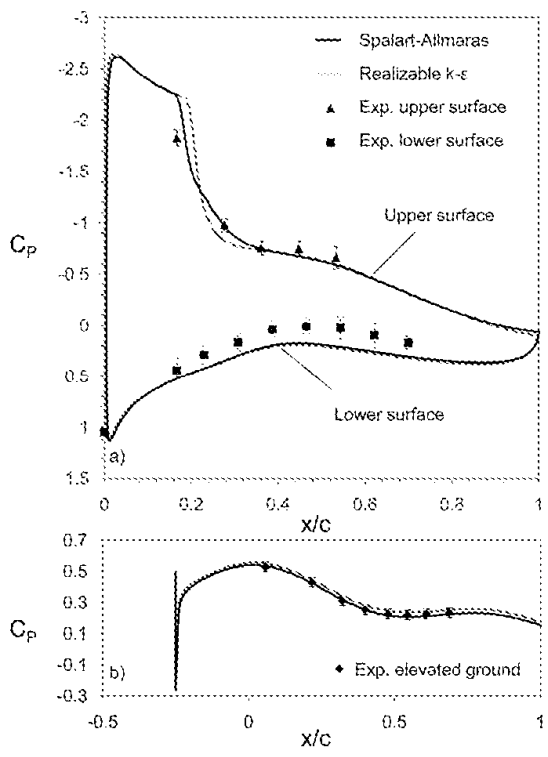

Figure 8. Chordwise pressure distributions with turbulence model comparisons to experiment for Case 2, for the semi-span (symmetry plane) on the wing (top), and elevated ground plane (bottom).

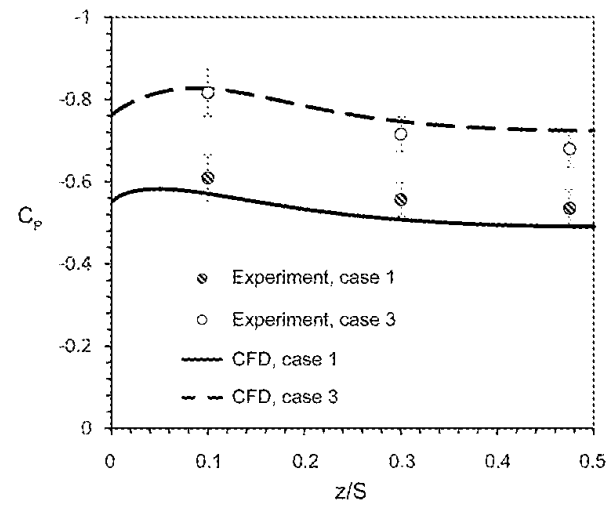

Figure 10. Spanwise pressure distributions at $x / c=0.17$ for cases 1 (steady state) and 3 (time-averaged unsteady). 
the leading edge until the shock/ground interaction point are predicted well by the SA model and poorly by the Realisable model, though at the shock location (which is time-averaged) it is more difficult to separate them as the motion of the shock causes a larger spread in the experiments than is represented in the numerical model.

On balance, it was decided that the Spalart-Allmaras model was providing a better match to experiments without the need for excessive mesh refinement, particularly in the presence of shock waves, and so was deemed to be the most effective model for the present research. Further experimental comparisons, including discussion of the influence of the model sting and additional examination of the consistency of the tunnel flow can be found in literature ${ }^{(20)}$.

\subsection{DISCUSSION}

\subsection{Quality of two-dimensional flow at the mid-span}

A wing aspect ratio of three does not guarantee two-dimensional flow at the semi-span, particularly in the presence of shock oscillation and uncertain transition behaviour ${ }^{(14,21)}$. The influence of spanwise flow is compounded in the present experiments by the flow around the endplate, which was observed in CFD to experience small-scale separation at the leading edge in some cases. As a rudimentary check of the quality of two-dimensional flow at the mid-span, comparisons were made to the three spanwise tappings at a chordwise location of $x / c=0 \cdot 167$ for cases 1 and 3 .

While the CFD shows good agreement with the experiments in terms of trends and actual values, the simulations indicate that while the flow at the mid-span was nominally twodimensional, the flow over the central third of the wing, where the staggered tappings were located, was not. As seen in Fig. 10, the variance between $z / s=0.33$ and 0.5 was several percent, and thus while the trends would not be markedly different, the quantitative values would have to be corrected using CFD as guidance if a more accurate two-dimensional plot were to be presented. For the present study, we are interested in the relative methods of ground representation, and thus some variance in spanwise pressure distribution is not a major consideration since both methods will experience the same issue and the numerical modelling replicates the experiment with reasonable fidelity. Blockage effects and a comparison of elevated ground and symmetry experimental methods

Data for cases 1 to 3 are presented with numerical comparisons in Fig. 11 as examples of the elevated ground and symmetry approaches, and as a means of exploring how influential the blockage effect was on the results obtained.

Although the freestream Mach number is only 0.531 for case 1 , CFD of the experimental setups for both elevated ground and symmetry methods reveal that the maximum local Mach number was relatively close to sonic. Considering first the experimental comparison, the increased Mach number over the upper surface of the wing in elevated ground mode implies that the effect of the ground boundary layer is to displace more air over the top of the wing, changing its effective angle-of-attack, as represented by the upper-surface pressure distribution in Fig. 11. The additional constriction of flow between the wing and the ground caused by the boundary layer is largely offset by this effect, such that the lower surface pressure gradient is reduced, resulting in a lesser suction peak at the point of minimum ground clearance. Although the CFD of the elevated ground appears to underpredict the suction peak by a $C_{P}$ of approximately $0 \cdot 15$, the general agreement for both sets of ground representations is relatively good. The experimental data indicates that around the lower-surface suction peak, the aerofoil 


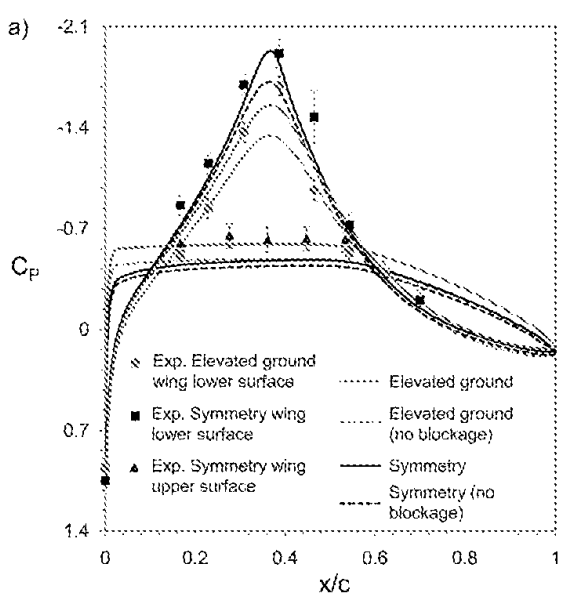

(a) Case 1

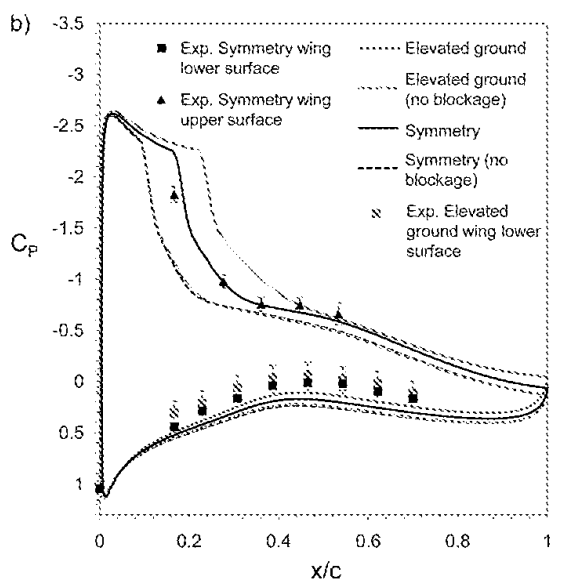

(b) Case 2

Figure 11. Chordwise pressure distributions with ground representation comparisons to experiment for: a) Case 1, b) Case 2, and c) Case 3, including cases run with negligible blockage.

over the elevated ground experiences a less significant negative $C_{P}$, with the greatest difference to the symmetry result coming behind the peak itself, suggesting a truncated region of acceleration.

The plot indicates that the influence of tunnel blockage on the results is considerable, and while simulations with negligible blockage produced the same trends, (increased flow over the upper surface, reduced lower suction peak), there was a clear offset in the pressure distributions due to the constriction of the flow imposed by the closed ceiling and floor. The effect on the upper surface was less pronounced, but the difference in lower surface $C_{P}$ was similarly significant compared to the experimental scenario.

Pressure distributions for Case 2 are shown in Fig. 11(b). Case 2 is at a higher angle of incidence, ground clearance and Mach number than Case 1, and a strong shock forms at around $x / c=0 \cdot 17$ with the symmetry method, very close to the first pressure tapping on the upper surface. The CFD was run as transient in the setup matching the experiment; a small separation bubble formed at the foot of the lambda shock which caused a mild unsteadiness in attempts to run the simulations as steady-state. Importantly, large-scale separation was present at the wing/endplate junction. The extent of separation at the wing/endplate junction was much greater in the elevated ground case, strongly influencing the rest of the flowfield and contributing to the differences observed, whereby the elevated ground case features a shock considerably further back on the wing. The additional flow being drawn over the wing due to the presence of the ground boundary layer slightly reduces the flow channelling between the wing and ground, causing a slight reduction in negative pressure compared to the symmetry case.

However, the comparison cases without blockage from the tunnel walls indicate that in that situation both ground representations produce near-identical results, highlighting a distortion of the experimental results caused by the proximity of the upper shock to the tunnel roof and the exaggerated effective angle of incidence that this produces around the wing. Without the constraints, the upper surface shock sits over $0 \cdot 1 c$ closer to the leading edge, though the flow is still separated at the shock foot. 


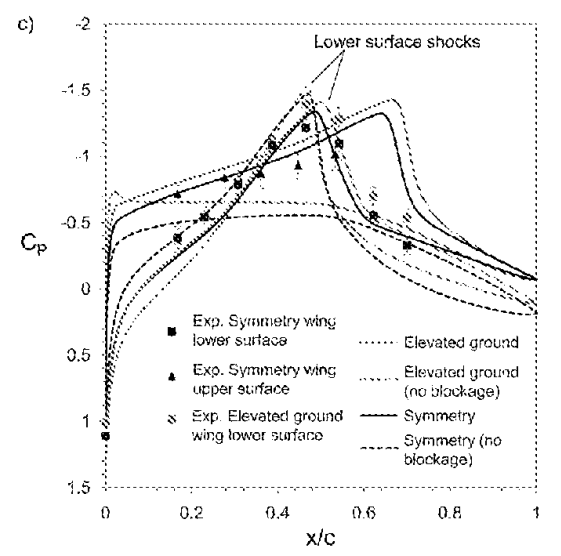

(c) Case 3
Case 3 was unique in that the flow was not steady and nominally two-dimensional at the mid-span, with the numerical results pointing to transverse flow fluctuations influencing the lower surface shock, which oscillated around an average location of approximately $x / c=0 \cdot 48$. The shock interaction at the wing surface is strong enough to separate the boundary layer in a periodic fashion. The upper surface shock was affected by the lower surface oscillations but it's movement was less pronounced around $x / c=$ $0 \cdot 7$. The flow at the wing/endplate junction was separated at the point of shock interaction, heavily influencing the flow to the trailing-edge and introducing the recurring spanwise disturbances. The pressure distributions are shown in Fig. 11(c).

Due to the influence of the elevated ground, the flow was more accelerated over both the upper and lower surfaces as compared to the symmetry case. The upper surface shock sits at an average of $x / c=0.03$ downstream of the location from the symmetry tests, and the lower surface shock sits $x / c=0 \cdot 02$ downstream in the same comparison. The lower surface shock in the elevated ground case forms a strong interaction with the ground boundary layer, which thickens markedly downstream of that point.

Most notably, however, the cases without tunnel blockage show that at this Mach number the flowfield does not produce an upper surface shock wave, and indeed the flowfield is steady-state, indicating that the transience observed in the tunnel simulations is induced by the proximity of the tunnel ceiling. There still exists a strong lower-surface shock, which sits further forward at approximately $x / c=0 \cdot 45$. For both the symmetry and the elevated ground case the shock strength is similar, though the pressure distribution behind the shock to the trailing edge is markedly different. A much greater proportion of negative pressure is generated over the upper surface in the elevated ground case, indicating a strong deflection of flow over the top of the wing due to the flow induced by the leading-edge of the ground plane.

\subsection{Two-dimensional numerical comparison of methods}

The comparisons in the previous section indicate that the tunnel data, while highlighting important differences between results produced by the two different ground representations, suffers from drawbacks stemming from blockage and endplate influence. While the data has been useful in validating the numerical method and providing preliminary insight into the performance of the two ground techniques, two-dimensional, steady-state and blockage-free results are presented here to provide an unambiguous comparison and analysis of methods for the cases examined.

Figure 12 presents Mach number contours around the section for symmetry and elevated ground as compared to an ideal moving ground case which will be assumed to be the 'correct' flowfield. Immediately noticeable is the small ground boundary layer which forms over the moving ground which cannot be reproduced in the symmetry case, but otherwise these two plots are highly similar. The elevated ground produces a much more pronounced 

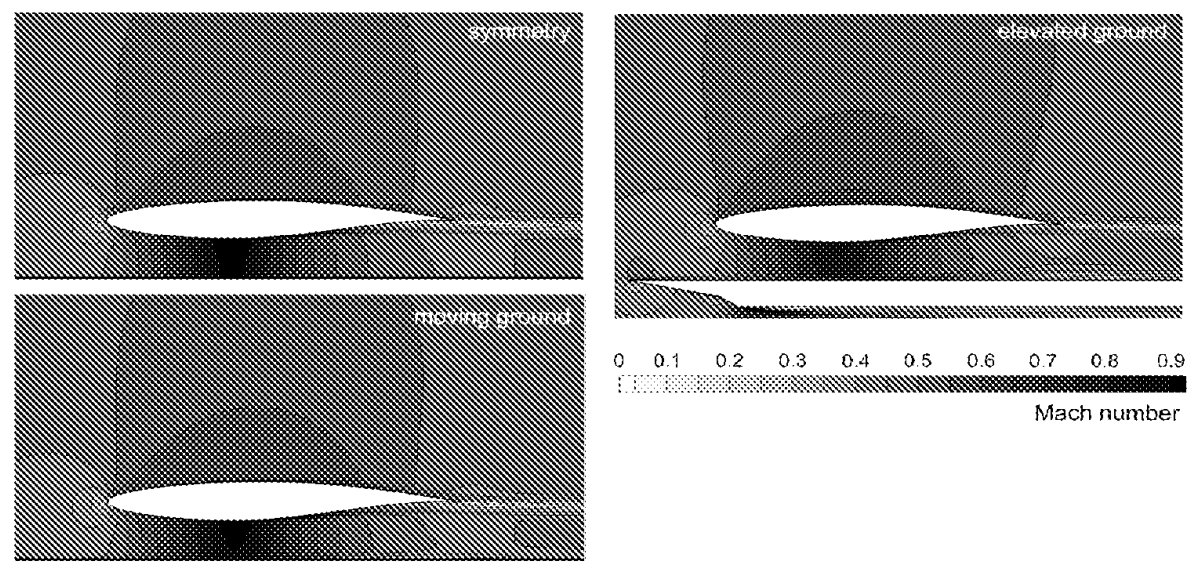

Figure 12. Case 1 comparisons; Mach number distributions for symmetry, elevated ground and moving ground representations.
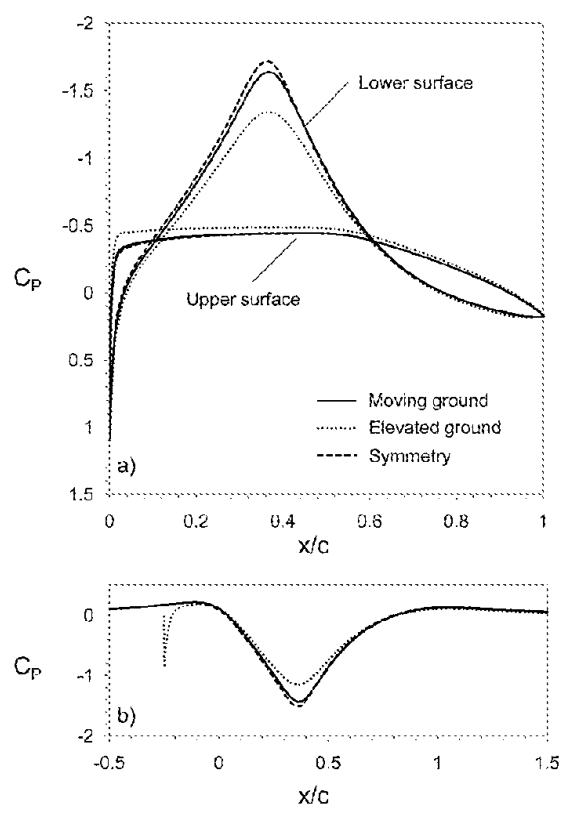

Figure 13. Pressure distribution at the mid-span symmetry plane for Case 1; comparisons of symmetry, elevated ground and moving ground representations. ground boundary layer, which is markedly thickened downstream of the high-speed region at the point of minimum clearance between the wing and ground. This influences the wake by deflecting it. The effect of the ground boundary layer and the influence of the ground plane leading edge has been to accelerate more flow over the upper surface, as previously discussed. Another influence is the subtle beginning of the merging of the region of flow near the leading edge, dominated by the stagnation zone, with its symmetric counterpart. It is this effect which has been shown to result in a recirculation near the leading edge at lower ground clearances and Mach numbers in literature ${ }^{(3)}$, but the experiments conducted here do not push into that territory.

The pressure distributions in Fig. 13 confirm that the symmetry and moving ground cases produce very similar results, with a mild over-prediction of negative pressure coefficient at the suction peak from the symmetry method, due to the lack of ground boundary layer. The elevated ground under-predicts the suction peak by a considerable margin, and the additional negative pressure over the upper surface is also 

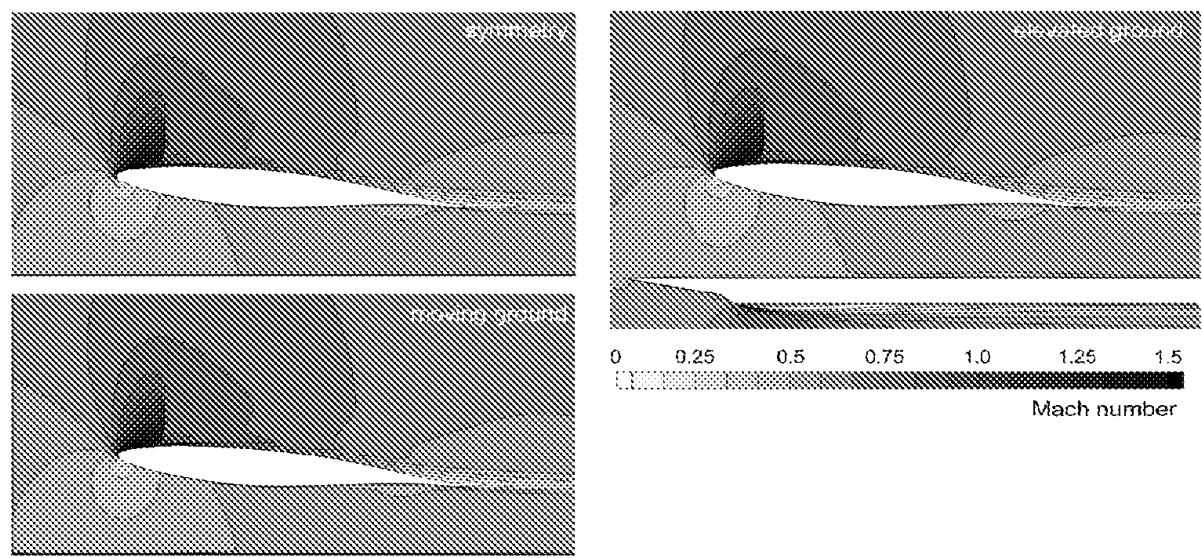

Figure 14. Case 2 comparisons; Mach number distributions for symmetry, elevated ground and moving ground representations.

notably different to the moving ground case. The effect on predicted lift is considerable; indeed it is conceivable, were this trend extended with a change to Mach number or ground clearance, that the elevated ground method could result in a prediction of net lift where the symmetry or moving ground cases would continue to predict a downforce.
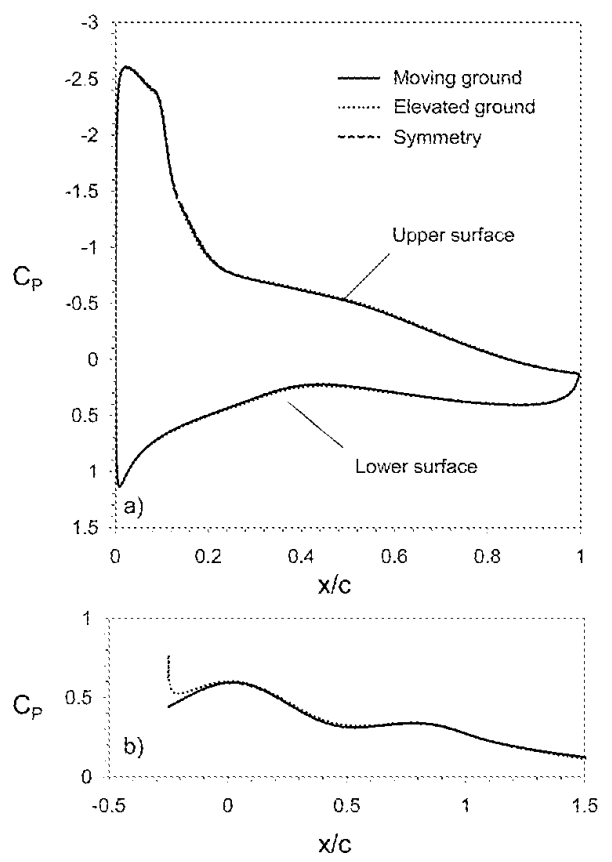

Figure 15. Pressure distribution at the mid-span symmetry plane for Case 2; comparisons of symmetry, elevated ground and moving ground representations.
The ground pressure distribution shows that the elevated ground quickly recovers from the leading-edge disruption, though the relatively thick boundary layer and the deflection of flow over the wing restricts the level of negative pressure generated under the wing and thus while the symmetry and moving ground results are highly similar, the elevated ground prediction is considerably worse.

Figure 14 presents Mach number contours for Case 2. The greater clearance and uppersurface shock, as previously discussed, mean that the ground is not such an important factor as with Case 1, and the two ground representations compare very well to the moving ground case. The small boundary layer on the elevated ground has little influence on the flowfield, and this is reflected in Fig. 15 where the pressure distributions around the wing and along the ground are near-identical for all three cases.

Figure 16 illustrates that although a strong shock has formed between the wing and ground (interacting with the ground at $x / c=0.55$ for symmetry and moving ground cases), the ground boundary layer is not a significant 

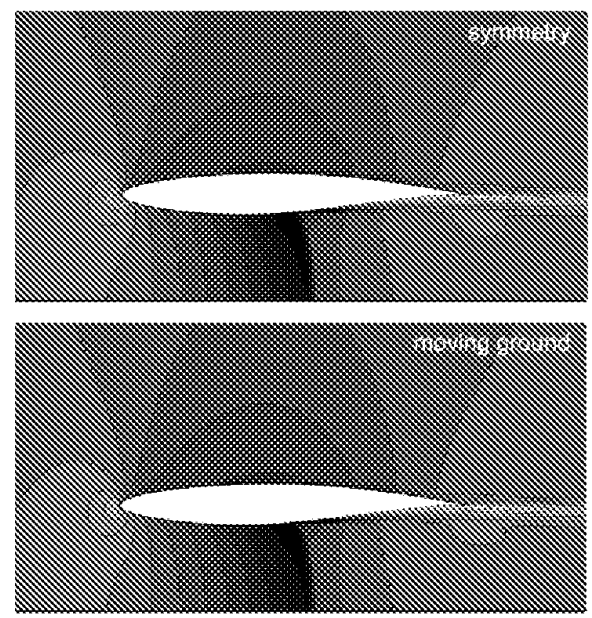

Figure 16. Case 3 comparisons; Mach number distributions for symmetry, elevated ground and moving ground representations.
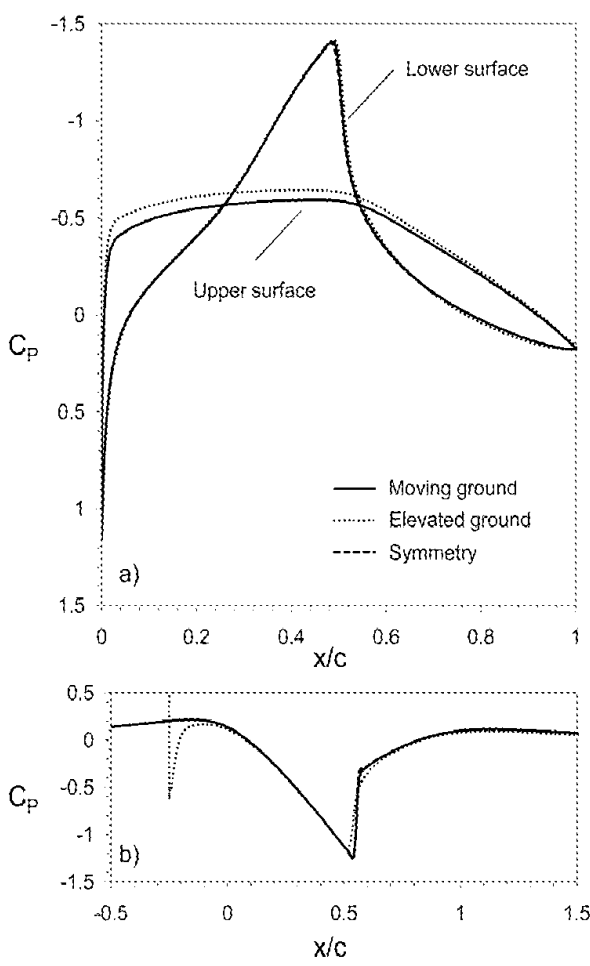

Figure 17. Pressure distribution at the mid-span symmetry plane for Case 3; comparisons of symmetry, elevated ground and moving ground representations.

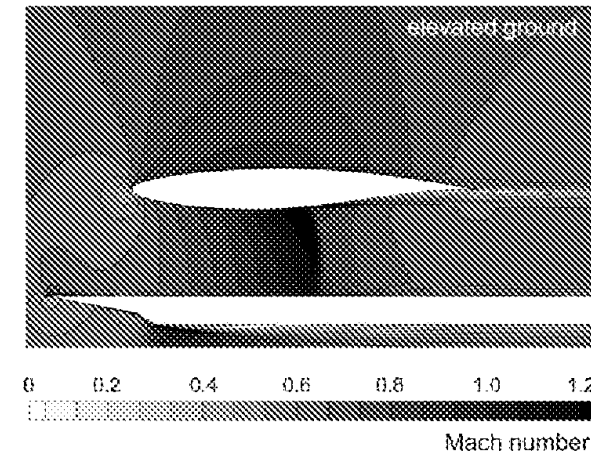

proportion of the channel. While the influence at the leading edge is considerable, the pressure field around the wing exerts a significant settling effect to squash the boundary layer against the ground. As reflected by the pressure distributions of Fig. 17 , the symmetry and moving ground plots match almost perfectly, whereas the elevated ground again promotes a slight increment in flow over the upper surface to increase the effective angle of attach. The more prominent ground boundary layer results in a less pronounced shock/ground interaction at $x / c=0.53$, with a cushioning effect seen to affect the shock foot implying a propensity towards a lambda structure. The downstream effect of this is such that the elevated ground pressure distribution does not recover to the same extent as the other two near-identical $C_{P}$ distributions, and would therefore exert a lingering influence into the wake region, particularly at very low ground clearances. At lower clearances and particularly in the presence of an oscillatory shock, this shock/ground interaction could potentially separate the ground boundary layer, which would have major implications for the accuracy of the rest of the flowfield. 
The primary conclusions from examining the three cases presented are that the ground representation is most critical when the ground clearance is low and when the wing is producing relatively little lift or downforce (i.e. close to neutral and sensitive to small changes). In all cases the symmetry method provided superior predictions of the pressure distributions which would be achieved were a moving ground possible, though in situations where the ground clearance is relatively high and the flowfield between the wing and ground does not feature a strong pressure gradient, the elevated ground provides satisfactory performance.

As discussed in previous sections, issues of blockage are critical when testing at this scale and while the symmetry method provides a better emulation of a moving ground in general, the need for effectively two models in the tunnel would become a limiting factor if the model was relatively large compared to the cross-section. In contrast, the elevated ground can remain relatively slim at greater scales, resulting in potentially much less blockage or the enabling of a larger test model. However, when the elevated ground itself reaches a critical Mach number, the resulting shock wave at the leading edge (where a separation bubble would usually occur) would significantly alter the flowfield that the wing would experience, adversely affecting the accuracy of the results.

\subsection{Further discussion}

The onset of supersonic flow around the lower surface of the elevated ground plane for Case 3 points towards a more concerning possibility: the emergence of a leading-edge shock structure on the ground plane. This would occur at Mach numbers slightly higher than those obtained in the windtunnel, but readily attainable in simulations. A strong, highly-disruptive shock can form close to the leading edge of the ground plane, which would clearly render the downstream flow as an inaccurate representation of the desired oncoming freestream conditions. It occurs because the elevated ground acts much as a wing would - the oncoming flow deflects and accelerates over the upper surface at the leading-edge separation bubble to the extent that the flow becomes supersonic well in advance of a supersonic freestream velocity. Furthermore this shock could well physically interact with the wing depending on its placement relative to the ground plane. Therefore, it is clear that the elevated ground cannot be applied even as a rough approximation of a moving ground at freestream Mach numbers which exceed the elevated ground's critical velocity.

The ground as designed for these experiments had a fairly simple, sharp leading edge. One can appreciate that even a better design of the leading edge, featuring a contour which avoids the risk of any separation, has two intrinsic limitations. Firstly it will accelerate the flow at the ground plane to a value which is faster than the freestream. This is particularly problematic if the ground clearance of the test model is small, in which case the lower surface could 'see' an entirely different oncoming flow than the upper surface. Secondly, introducing significant curvature at the leading edge, even if it is designed to settle the flow downstream to a level which would not significantly disturb the wing, would still result in the emergence of a shock wave at a certain early critical Mach number. If any form of suction or blowing were to be used to minimise the ground boundary layer, the disturbances introduced into the flowfield by the apparatus, including small shock waves, could prove prohibitively disruptive at low clearances. The symmetry method suffers from none of these drawbacks.

There are also issues of scale to consider in conducting tests such as these. The scaling of tests from wind-tunnel to actual flight is of course a long-standing challenge in aeronautics ${ }^{(21)}$, and, at small-scale, is somewhat enhanced for ground effect problems due to the relatively large influence of the body boundary layer size with regards to the ground clearance. If the elevated ground is used, its boundary layer contributes further to this problem, as we have already seen. 
At Reynolds numbers of higher orders of magnitude, the boundary layers are markedly smaller in relation to the body, and thus the effective channel created by the object and the ground is altered. At this stage, if an elevated ground is used and is of sufficiently good design to provide smooth flow that is as unperturbed at the leading edge as possible, the boundary layer influence on the size of the gap between object and ground will be reduced to the point at which it will not have a marked influence. Thus at a larger scale a well-designed elevated ground plane may prove preferable for testing up until the point at which the flow close to the leading edge becomes near-sonic.

Assuming the higher Reynolds number is achieved by directly scaling up the model, the symmetry method can suffer from a large blockage ratio compared to an elevated ground which can remain fairly slim as it is scaled up despite having to span the test section. Thus the choice of method for testing may even depend on the size of the test section, at least up until near-sonic freestream Mach numbers where the symmetry method is the only one which could be relied upon.

\subsection{CONCLUSIONS}

Two methods of investigating ground effect problems at high subsonic Mach numbers have been proposed as an inexpensive and effective alternative to a moving ground. Experiments were conducted in a relatively small transonic blowdown tunnel using the RAE2822 section as an example geometry at Reynolds numbers of approximately $0 \cdot 8 \times 10^{6}$. Numerical analysis was used to complement the pressure coefficient results to provide significant additional insight into the flows observed and allow a direct comparison to moving ground simulations. The two methods investigated were an elevated ground plane and a symmetry (or mirror-image) approach, and were tested in different combinations of freestream Mach number and ground clearance. The main conclusions can be summarised as follows:

- For all Mach numbers and ground clearances investigated, the symmetry method provided a superior match to results obtained from CFD featuring a moving ground. Only at clearances approaching a height-to-chord ratio of 0.1 do minor discrepancies in the pressure distribution close to the leading edge and suction peak appear. This is partly due to the minor influence of the thin ground boundary layer forming on the moving ground but not on the symmetry axis, and also due to discrepancies emerging as the region close to the stagnation zone begins to merge with the ground and symmetry plane, behaving differently in each case.

- The elevated ground has a tendency to increase the effective angle-of-attack of the wing by deflecting more air over the upper surface. At low ground clearances, the ground boundary layer serves to further constrict the flow between the wing and ground such that higher Mach numbers can be achieved over both surfaces of the wing compared to those obtained with the symmetry method. Both these effects will serve to reduce the critical Mach number and hasten the onset of the buffet boundary as a result.

- The elevated ground approach has an in-built limitation in that when it reaches its own critical Mach number, the downstream flow would be significantly disrupted and bear little resemblance to that which would be attained with the symmetry method.

- Issues of scale are present as they would be in any wind-tunnel test, however complicated slightly by the relatively large boundary layer compared to the overall ground clearance.

The effectiveness of the approach was proven despite the limitations of the experimental 
design including significant interference from the endplates and relatively low-resolution pressure data as an output. If a testing programme were to be devised to investigate a similar problem thoroughly, it would be vital that the wing was of higher aspect ratio and the wall interference was reduced, and transition-fixing would be preferable. In this instance the porous floor and ceiling of the tunnel were closed off to facilitate ease of numerical reproduction of the apparatus, but blockage effects would be dealt with much more effectively if relaxation of the flow was permitted. Nevertheless, the preliminary experimental results and accompanying numerical analysis indicates that the symmetry method is generally preferable as a technique for such flows whenever practical to implement.

\section{ACKNOWLEDGEMENTS}

The authors gratefully acknowledge the support of The Royal Aeronautical Society: travel by Graham Doig to the US Naval Academy to collaborate on the research in 2008 was made possible thanks to the RAeS through a Centennial CAARC Award to the primary author.

\section{REFERENCES}

1. STRIKE, W.T. and LUCAS, E.J. Evaluation of wind tunnel tests on AFMDC Monorail cone and spike nose sled configurations at Mach numbers from 2.0 to 5·0, 1968, AEDC-TR-68-198 (AD679206).

2. TORDA T.P. and UZGIRIS S.C. Blue flame - A land speed record vehicle, Mechanical Engineering, 1970, 92, (7), pp 9-18.

3. Goldberg, U., Akdag, V., Palaniswamy, S., Oberoi, R., Bachchan, N., Glessner, P. and Fredrick, W. CFD Analysis of the American Challenger Rocket Car, 2006, SAE 2006 World Congress \& Exhibition (Vehicle Aerodynamics), pp 251-258.

4. WEISELSBERGER, C. Wing resistance near the ground, 1922, NACA TM-77.

5. BARBER T.J., LEONARDI E. and ARCHER, R.D. Causes for discrepancies in ground effect analyses, Aeronaut J, 2002, 106, (1066), pp 653-657.

6. FINK, M.P. and LASTINGER, J.L. Aerodynamic characteristics of low-aspect-ratio wings in close proximity to the ground, 1961, NASA Technical Note D-926.

7. AHMED, M.R. and SHARMA, S.D. An investigation on the aerodynamics of a symmetrical airfoil in ground effect, Experimental Thermal and Fluid Science, 2005, 29, pp 633-647.

8. Sowdon, A. and HoRI, T. An experimental technique for accurate simulation of the flowfield for wing in surface effect craft, Aeronaut J, 1996, 100, (996), pp 215-222.

9. SkEws, B.W. Three-dimensional effects in wind tunnel studies of shock wave reflection, $J$ Fluid Mechanics, 2003, 407, pp 85-104.

10. Schmisseur, J.D. and Gaitonde, D.V. Numerical investigation of strong crossing shockwave/turbulent boundary-layer interactions, AIAA J, 2001, 39, (9), pp 1742-1749.

11. Doig, G., Barber, T.J., Leonardi E., Neely, A.J. and Kleine, H. Methods for investigating supersonic ground effect in a blowdown wind tunnel, Shock Waves, 2008, 18, (2), pp 155-159.

12. Aeschliman, D.P. and OBERKAMPF, W.L. Experimental methodology for computational fluid dynamics code validation, $A I A A J, 1998,36$, (5), pp 733-741.

13. Cook, P.H., McDonald, M.A. and Firmin, M.C.P. M.C.P., Aerofoil RAE 2822 - Pressure Distributions, and Boundary Layer and Wake Measurements, 1979, Experimental Data Base for Computer Program Assessment, AGARD Report AR 138.

14. Suddani, N., SATo, M., Kanda, H. and MAtSuno, K. Flow visualization studies on sidewall effects in two-dimensional transonic airfoil testing, J Aircr, 1994, 31, (6), pp 1233-1239.

15. Fluent User Guide, 2006, FLUENT Inc., Lebanon, NH.

16. SutherLAND, W. The viscosity of gases and molecular force, Philosophical Magazine, 1893, 5, (36), pp 507-531.

17. Spalart, P. and Allmaras, S. A one-equation turbulence model for aerodynamic flows, Recherche Aerospatiale, 1992, 1, pp 5-21. 
18. MeNTER, F.R., LANGTRY, R. and VöLKER, S. Transition modelling for general purpose CFD codes, flow, Turbulence and Combustion, 2006, 77, (1-4), pp 277-303.

19. SHIH, T.H., LIOUZ, W.W., SHABBIR, A., YANG, Z. and ZHU, J. A New $k-\varepsilon$ Eddy-viscosity model for high Reynolds number turbulent flows - Model development and validation, Computers \& Fluids, 1995, 24, (3), pp 227-238.

20. DoIG, G. Compressible Ground Effect Aerodynamics, PhD Thesis submitted to the University of New South Wales, Sydney, Australia, 2009.

21. GarbaruK, A., Shur, M., Strelets, M. and Spalart, P.R. Numerical study of wind-tunnel wall effects on transonic airfoil flow, ALAA $J, 2003,41$, (6), pp 1046-1054.

22. BushnELL, D.M. Scaling: wind tunnel to flight, Annual Review of Fluid Mechanics, 2006, 38, pp 111-128. 